\title{
Molecular Identification of Fusarium proliferatum from toenails: A case report
}

\author{
Sazan Jamal Gharib \\ Medical Laboratory Department \\ Sulaimani Technical Institute \\ Sulaimani Polytechnic University \\ Zakho University \\ Sulaimani, Iraq \\ Sazan.gharib@spu.edu.iq
}

\author{
Nawroz Abdul-razzak Tahir \\ Horticulture Department \\ College of Agricultural Sciences, \\ University of Sulaimani \\ Sulaimani, Iraq \\ Nawrozbiology@gmail.com
}

\begin{abstract}
Volume 4 - Special
Issue: 3rd International Conference on Health \& Medical Sciences: Insight into Advanced Medical Research

(ICHMS 2019)
\end{abstract}

\section{DOI:}

10.24017/science.2019 .ICHMS.13

Received:

9 June 2019

Accepted:

6 July 2019
Abstract

Fusarium is a saprophytic fungus which is broadly disseminated in soil, plant debris and other organic substrates. These fungi can affect indigenous tissue in immunocompromised patients and result in some fungal infections such as Onychomycosis, bone and joint disease, or sinusitis. In this paper, we report a 56-year-old female has Diabetes milletus who established to disseminate Fusarium infection in her left first toenail from the Dermatology Department / Khabat Skin Center, Sulaimani Province, Iraq. To study of these fungi as the true causative agents of nail infection, we describe a PCR/sequencing assay to confirm routinely diagnosis of the infecting fungi in this case with toenail Onychomycosis. Designation of the ordinary laboratory of Fusarium is very challenging because of the septate hyphae of Fusarium are hard to discriminate from those of Aspergillus, which has a more favourable consequence and this time-consuming. Therefore, molecular tools were used for identification of $F$. proliferatum from this case by amplifying ITS region of rDNA using a pair of universal primers ITS1 and ITS4. Infectious fungal molecular detection advances the select of right antifungal therapy, in that way improving the medication rate of nails infections. DNA sequence analysis and Phylogenetic tree of the amplified ITS-rDNA was used for species identification of this strain of $F$. proliferatum. For final identification sequencing of DNA has been carried out by [Macrogen Korea] using forward primer ITS1, The obtained consensus of query sequence was compared with the ITS DNA database on the BLAST homepage, aligned and diagnosed strain deposited to the GenBank and we received an accession number (MK112619). The sequence of the $F$. 


\begin{abstract}
proliferatum amplicon was aligned using ClustalW and the alignment was used to make phylogenetic analysis using MEGA software version $X$. To the best of our knowledge, the isolated Fusarium in this paper is the first case of its kind to be sequenced and reported by the molecular method in Kurdistan region of Iraq
\end{abstract}

Keywords: Fusarium proliferatum, Toenail, Onychomycosis, PCR, ITS region, Iraq.

\title{
1. INTRODUCTION
}

Nail infection (Onychomycosis) due to fungi is a communal disease of the fingernails or toenails of dermatophytes, yeasts, and non-dermatophytic molds. Today, Onychomycosis is a major public health case due to its growing incidence and has considerable clinical implications as well as helping as an illness reservoir [1]. The occurrence of nail infection has been described to be 23\% through Europe [2] and 20\% in East Asia [3]. In North America, the prevalence of onychomycosis is up toward 14\% [4], with fungaldisease accountable for $50 \%$ of all nail disease [5]. Nail diseases come to be more public with growing oldness and generally toenails are impacted ( $80 \%$ of cases).

Nail trauma, frequent water immersion, occlusive footwear, athlete's foot, diabetes mellitus, immunosuppression and smoking are the principal deterrent determinants for onychomycosis [6]. Other susceptibility variables including increased occurrences of nail disease such as decreased peripheral mobility, diabetes, nail trauma, and bad nail hygiene were also worried. However not life-threatening, it can have important clinical implications, like secondary bacterial diseases, chronicity, therapeutic problems and disfiguration, besides serving as an illness reservoir [7].

Molds are soil-living saprophyte fungi and some are pathogens. They have commonly been deemed to contaminate the skin and nails ' fungi or secondary pathogens, but some of them may be main contaminants e.g., Scopulariopsis brevicaulis, Fusarium sp, Aspergillus sp., Scytalidium hyalinum and, recently, Onychocola canadensis [8,9,10,11,12]. Fusarium spp. is a leading cause of human infections, especially in immunocomporomised patients [13]. The pathogen frequently infects soft tissues by traumatic inoculation in ordinary hosts [14]. The types of these diseases are onychomycosis [15], keratitis and endophthalmitis [16], and mycetoma [17].

Fusarium is a non-dermatophytic mould found in the form of soil saprophytes and pathogens of plants. Human illnesses are likely the consequence of different catalyzing variables of deficient immune status. Older-age immunocompetents are also susceptible to multiple unassumed saprophytic and plant pathogens. It is therefore of utmost significance that this newly evolving fungal pathogens be properly identified and that adequate therapy be instituted as soon as possible to control human infections so that spread infections can be prevented [18].

In the current study, we reported a case of Onychomycosis in a left first toenail of a 56-yearold female with a chronic toenail lesion caused by a non-dermatophytic mold, Fusarium proliferatum, a saprophytic organism commonly found in soil, plant debris and other organic materials that can induce local tissue infections in diabetic patients like onychomycosis. A mixture of morphology, molecular and phylogenetic research disclosed it was F. proliferatum [19]. 


\section{CASE PRESENTATION}

A 56-year-old female with chronic Diabetes mellitus, from Khabat Skin Center in March 2017with greenish discoloration and disfigurement in her great left toenail, had depressed nail plate and interdigital maceration, no itching pain and discharge were noted (Fig.1: A and B). There was a history of probable Onychomycosis on her both toenails (not verified by mycological examination) two years previously (in 2015), that completely disappeared after treatment with antifungal therapy. On examination, left great toenail was green to light yellow in color and there were dystrophic changes in the nail plate that toenail (Fig.1). The associated factor for this study case was previously diagnosed (of 4 years duration) diabetes mellitus, and at the time of sampling, the patient had undergone diabetes medication. $\mathrm{KOH}$ examination and LPCB stain from the nail clipping sample from this study case revealed numerous hyaline branched and septate hyphae and Chlamydospores provocative reproductive constructions. A culture on SDA at $30^{\circ} \mathrm{C}$ (room temperature) from the left toenail grew Fusarium proliferatum and the patient was settled on oral Itraconazole $200 \mathrm{mg} /$ day and advanced to use it for at least 3-4 months.

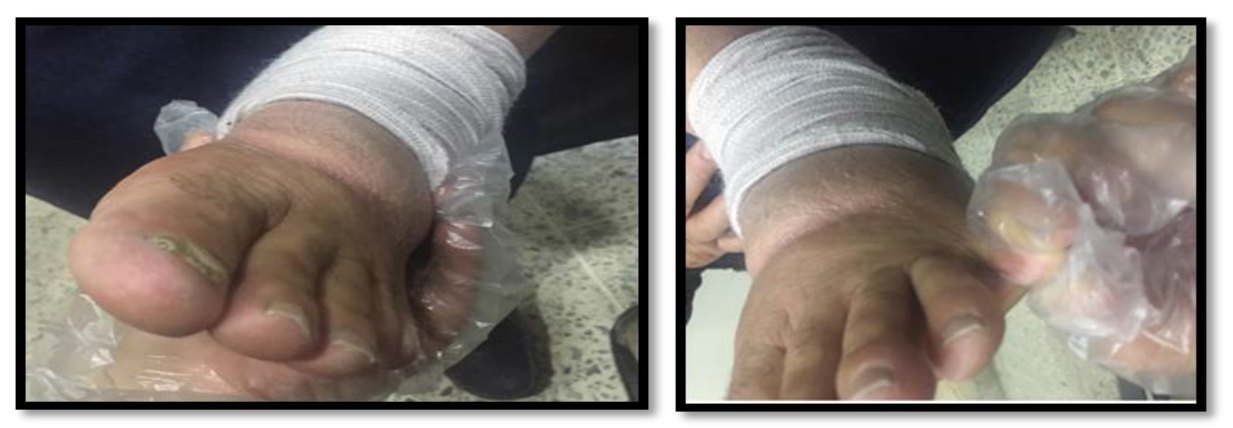

Figure 1: The First toenail of the left feet of 56 years-old woman showing depressed nail plate and extensive greenish discoloration in here left the first toenail due to Fusarium proliferatum.

\section{METHODS AND MATERIALS}

\subsection{Sample Collection, Processing and Cultivation on Selective media}

Specimen of nail clipping from the affected nail was collected after proper sterilization of the affected nail with $70 \%$ alcohol. The sufficient amount of sample was transported in the folded dry square of the paper envelope to the mycology research laboratory in Zakho University / Faculty of Science/Biology Dept. quickly for mycological examination [20,21].The mycological studies were performed in Mach 2017. Sample obtained from the toenail clipping was divided into two portions; one portion was placed on the surface of a clean slide, observed through direct microscopic examination after mounting in 1-2 drops of $20 \% \mathrm{KOH}$ solution (Merck, Germany), covered with a clean cover slip, the slide was passed through the burner flame several times (the $\mathrm{KOH}$ will digest the host material and keratin and also clear the fungal elements) [22], the process is speeded up by gently heating , then incubated for a 5-30 min., finally each slide was examined by using light microscope (CX 40 Olympus USA) for the presence of fungal (hyphae, transversally septate macro and/or micro conidia and Chlamydospore) [23].

Direct microscopy for this case was positive (found branched septate hyphae). The remaining part of the nail cuts were also grown on two sets of dextrose agar from Sabouraud (SDA) medium (CM41; Oxoid, UK): one plate of SDA with antibiotics and cycloheximide) [SigmaAldrich] (500 mg/l), and one slant of SDA contained antibiotics but without cycloheximide, 
which was incubated at $30^{\circ} \mathrm{C}$ (room temperature) for 2-3 weeks. After 5 days, growth was seen on both plate and slant cultures in the form of white, cottony colonies which later turned pink in color (Figure 2). There has been no detection of bacteria growth in culture. Detailed research of their colonial characters and microscopic morphology in lacto phenol cotton blue (LPCB) mounts has recognized the extracted fungal isolate to species level as far as possible. Primary isolation of the fungus was depended on morphological characteristics of the culture (Macroscopic and Microscopic feature of the colonies), colonies were examined microscopically for identification of (existence, shape and arrangement) of irregular branched septate hyphae, and sickle-shaped macroconidia and other structures needed for diagnosis of this genus such as septate microconidia and chlamydospore (Figure 3). Contaminant molds were excluded during the microscopic examination, while diagnosed species of Fusarium isolates were sub cultured and purified again in a new agar slant of SDA and maintained for further confirmation by molecular method.

\subsection{Molecular Studies \\ 3.2.1 Isolation of Fungal DNA}

Fungus DNA was isolated and purified via taking a proper amount from the fresh colony (21 day-olds colony grown on SDA medium) by grinding in existence of liquid nitrogen for first braking up of mycelia. Total DNA extraction of fungi by molecular biology kit (EZ-10 Spin Column Fungal Genomic DNA Mini-preps kit) was isolated as suggested by the manufacturer. Phenol-chloroform was used formerly for DNA isolation but was substituted as a kit by the quicker and environmentally friendly product. To shatter the cell wall and cell membrane, the mycelium had to be powdered before the lysis move. About 100mg (approximately 5 cubic $\mathrm{mm}$ ) [24] of fungal tissue (mycelia/spores) on SDA was weighted out in a mortar, grinded with a pestle to dust and thaw with liquid nitrogen. Transfer grinded sample to a clean $1.5 \mathrm{ml}$ microtube containing $180 \mu \mathrm{l}$ Universal Digestion Buffer, add $20 \mu \mathrm{l}$ Proteinase K to the specimen and blend carefully by stirring and incubation for $60 \mathrm{~min}$ at $56{ }^{\circ} \mathrm{C} .100 \mu \mathrm{l}$ Universal of Buffer PF was added, mix, then inverting, and incubate at $-20^{\circ} \mathrm{C}$ for 5 minutes. Centrifuge at $12,000 \mathrm{x}$ g for 5 minutes at room temperature. Transfer the supernatant to a new $1.5 \mathrm{ml}$ tube.5. Add 200 Universal Buffer BD, mixes thoroughly by vortexing. Add $200 \mu 1$ ethanol $(96$ $\%)$, mix well by vortexing. Transfer the combination to the column EZ-10 in a reserve tube of $2 \mathrm{ml}$. Centrifuge at $(12,000 \mathrm{rpm})$ for $1 \mathrm{~min}$. Discard the flow-through. Add $500 \mu \mathrm{l}$ Universal PW Solution, and centrifuge for 60 seconds at (12,000 rpm). Discard the flow-through. Add $500 \mu \mathrm{l}$ Universal Wash Solution, and centrifuge for $1 \mathrm{~min}$ at $(12,000 \mathrm{rpm})$. Reject the flowthrough. To dry the EZ-10 membrane, the empty column puted in the micro centrifuge and spin for a $2 \mathrm{~min}$ at (12,000 rpm). Discard the supernatant and it to a clean $1.5 \mathrm{ml}$ centrifuge tube. Then add $100 \mu \mathrm{l}$ Buffer TE directly onto the EZ-10 membrane. Incubate at room temperature for $1 \mathrm{~min}$, centrifugation for $1 \mathrm{~min}$ at $(12,000 \mathrm{rpm})$ to elute the DNA. The DNA sample was kept at $4^{\circ} \mathrm{C}$ for further use. Purified genomic DNA arranges 20-50kb in length (www.bioneer.com).

\subsubsection{Detection of Genomic DNA}

The concentration and purity (quantitative estimation) of the fungal DNA was completed by agarose gel electrophoresis and Nano drop UV spectrophotometer at $2601280 \mathrm{~nm}$. To detect the presence of genomic DNA $6 \mu \mathrm{L}$ of the fungal DNA sample was mixed with $1 \mu \mathrm{L}$ of loading dye and loaded in the agarose gel (1.5\%). The gel was run at 90 volts for $45 \mathrm{~min}$. until the loading dye migrated to the suitable distance from the well then, the gel was examined by gel image desktop to see the DNA band was visualized under Ultraviolet Trans illumination [25]. And the purity and concentration of genomic DNA measured by dispensing $1 \mu \mathrm{L}$ from the DNA specimen on the lower optical pedestal after cleaning and calibration by deionized distilled water the lever arm was closed and selected (Measure). The software automatically measured the concentration and purity of nucleic acid specimen [26]. 


\subsubsection{PCR and Primer preparation}

The ITS1 and ITS4 primers were provided by \{MWG-Biotech AG Germany\} in lyophilized form, dissolved in sterilized deionized distilled water to obtain $10 \mathrm{Pm}$ as a final concentration and stored in the deep freezer until using [27] as shown in Table (1). The PCR master mix contained all the components needed for the PCR reaction except DNA template and primer, it containing (2X Master mix) which containing: prime Top DNA polymerase (1U), $1.5 \mathrm{mM}$ $\mathrm{MgCl}$, reaction buffers (pH 8.5), $250 \mu \mathrm{M}$ of each dNTP (dATP,dCTP,dGTP and dTTP) and stabilizer and tracking dyes which function as loading dyes. PCR reactions were carried out in (Thermocycler, Eppendorf) PCR machine, components requirements for PCR reaction are provided in Table (2). Thermo cycling program for isolated fungus was as following conditions: An first denaturation $\left(94^{\circ} \mathrm{C}\right.$, for 5 min.) ,then 35 cycles of denaturation $\left(94^{\circ} \mathrm{C}, 1\right.$ min.), annealing $\left(58^{\circ} \mathrm{C}, 1 \mathrm{~min}.\right)$ and extension $\left(72^{\circ} \mathrm{C}, 1 \mathrm{~min}.\right)$, with a final extension step $\left(72^{\circ} \mathrm{C}, 10 \mathrm{~min}\right.$.), Table (3).The PCR products were analyzed by electrophoresing $10 \mu \mathrm{l}$ of amplicons in $2 \%$ agarose gel in TBE buffer , dyed with bromide of ethidium and provided for 90 minutes at 75 volts. The gel was visualized and photographed under a computerized UV trans-illuminator. Undigested (unrestricted) $\lambda$ DNA (100 bp) was used as a reference to conclude the size of fragments.

Table 1: Oligonucleotide primers used in the PCR reaction.

\begin{tabular}{|cc|}
\hline Primer & \multicolumn{1}{c|}{ Sequence $\mathbf{5}^{\mathbf{}} \boldsymbol{\rightarrow} \mathbf{3}^{\mathbf{\prime}}$} \\
\hline ITS1 (forward) & TCCGTAGGTGAACCTGCGG \\
\hline ITS4 (reverse) & TCCTCCGCTTATTGATATGC \\
\hline
\end{tabular}

Table 2: Components required for PCR reaction.

\begin{tabular}{|ccc|}
\hline Components & Volume $(\boldsymbol{\mu l})$ & Concentration \\
\hline 2X Ready master mix & $5 \mu$ & 1 X \\
\hline Forward primer & $2 \mu \mathrm{l}$ & $10 \mathrm{pmol}$ \\
\hline Reverse primer & $2 \mu \mathrm{l}$ & $10 \mathrm{pmol}$ \\
\hline Template DNA & $5 \mu \mathrm{l}$ & $<250 \mathrm{ng}$ \\
\hline Deionized water & $11 \mu \mathrm{l}$ & \\
\hline Total volume & $25 \mu \mathrm{l}$ & \\
\hline
\end{tabular}

Table 3: PCR program for amplification by ITS1 and ITS4 primers.

\begin{tabular}{|lcc|c|}
\hline Steps & Temperature & Time (min.) & Number of cycles \\
\cline { 1 - 2 } Initial denaturation & $94^{\circ} \mathrm{C}$ & 5 & 1 \\
\hline Denaturation & $94^{\circ} \mathrm{C}$ & 1 & \multirow{2}{*}{35} \\
\hline Annealing & $58^{\circ} \mathrm{C}$ & 1 & \\
\hline Extension & $72^{\circ} \mathrm{C}$ & 1 & 1 \\
\hline Final extension & $72^{\circ} \mathrm{C}$ & 10 & \\
\hline
\end{tabular}

\subsubsection{Sequencing, Multiple alignment and Phylogenetic Tree}

In rDNA the gene of Internal transcribed spacer region was amplificated by using ITS1 and ITS4 primers and caused in PCR pattern of 471 bp for Fusarium oxysporum. The ITS1 fragment from this strain of Fusarium in the current study, which preliminary amplified using ITS1 and ITS4 primers were sequenced using only forward primer ITS1 and 10 $\mu$ of purified PCR amplicon according to Macrogen Company/Korea, by using automated DNA sequencer. Alignment and checking of the obtained query sequences were conducted using BioEdit Sequence Alignment Editor 7.0.5.3 (http://www.mbio.ncsu.edu/BioEdit/bioedit.html). For final diagnosis confirmation, the obtained consensus of query sequence was compared with the 
ITS DNA database. The recent sequences have been deposited (MK112619) in GenBank Table (4). Sequence of the isolated fungus was aligned using ClustalW and MEGA software version X. Phylogenetic analyses using the Neighbour-Joining (NJ) method [28] was performed for this strain with the MEGA version X software using p-distance model [29] with the 'pairwise deletion of gap option'. The robustness of the branches were assessed by bootstrap analysis with 1000 replicates.

\section{RESULTS}

\section{A. Primary Isolation and Culture processing}

Mycological examination relies on species level is very important for the choice of best therapeutic agents. Based on clinical examination, a 56-year-old diabetic woman showed the evidence of toenail Onychomycosis who's screened for nail diseases or dermatomycoses as a case report in this study, in March 2017. In the mycological test of the case, which included direct $\mathrm{KOH}$ examination before specimen cultured, considered positive for the left toenail, due to evident of numerous hyaline branched and septate hyphae and reproductive structures suggestive of chlamydospores. While the white, cottony causative agent after 5 days incubation on SDACC at $30^{\circ} \mathrm{C}$ was Fusarium proliferatum (Figure 2). The genus and species diagnosis confirmed after staining with LPCB stain; irregular branched septate hyphae, sickleshaped hyaline septate macroconidia, few microconidia and pyriform chlamydospore were observed under a light microscope. The sequencing of the ITS-region of the isolated strain from this clinical specimen confirmed isolation of the same fungus in this case study.

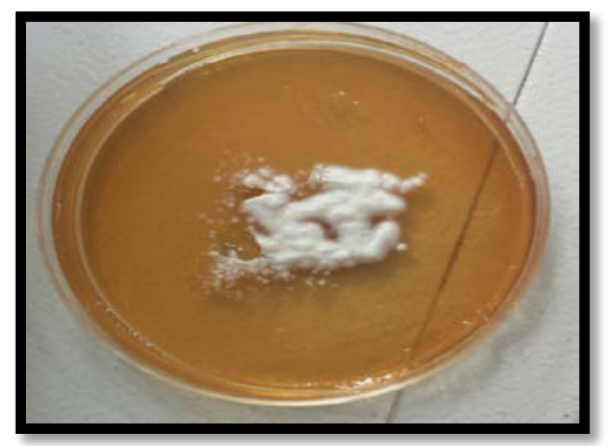

Figure 2: Colonial morphology of $F$. proliferatum after three weeks of incubation at $30^{\circ} \mathrm{C}$ on SDA.

\section{B. Molecular Identification}

After phenotypic diagnosis, we applied the PCR and sequencing technique successfully for the investigated strain of $F$. proliferatum using universal fungal primers for amplifying ITS region of rDNA as a molecular tool. Fungus genomic DNA was extracted from isolated fungus using liquid nitrogen and molecular biology kit (EZ-10 Spin Column Fungal Genomic DNA Minipreps Kit, Canada). Fungus DNA was observed by electrophoresis on 1.5\% agarose gel. The ITS region on rDNA was amplified by by means of universal primers ITS1 and ITS4 which yielded in PCR product size of $471 \mathrm{bp}$ for the studied isolate, as shown in (Figure 3). Recognition pattern on agarose gel $2 \%$, later confirmed by sequence analysis, Table (4). The current practical PCR finding was sequenced using only forward primer ITS1 on automated sequencer according to (Macrogen Company, Korea) and comparison of the obtained sequence with the other Fusarium species ITS-DNA on BLAST search tool home page.

Alignment of the obtained sequence of the isolated strain of the genus in this study plus the reliable GenBank sequence confirmed the fact that different species of non-dermatophytic moulds have different ITS1 and ITS2 sequence, make the ITS region as a good target for post 
PCR manoeuvres such as sequencing. The obtained sequence was deposited in GenBank and we received accession number, Table (4).

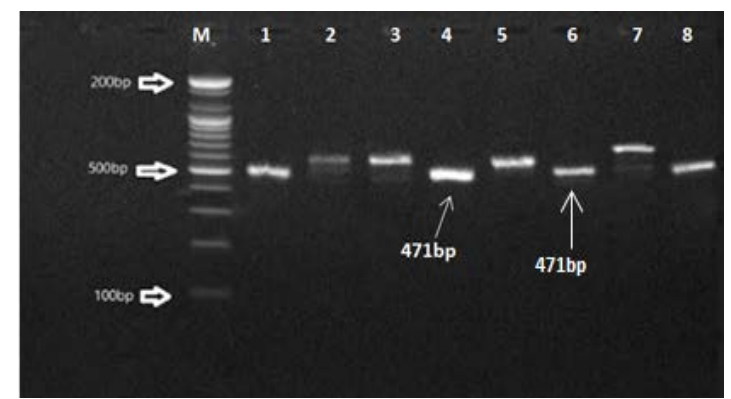

Figure 3: Agarose gel electrophoresis (2\%) of ITS-PCR products of F. proliferatum (Lane 6), M: 100bp molecular size marker.

Table 4. Fragment size of PCR-ITS region in tested $F$. proliferatum.

\begin{tabular}{|l|cc|}
\hline Isolate & $\begin{array}{c}\text { Size of PCR- ITS (bp) } \\
\text { F. prolieratum }\end{array}$ & $\begin{array}{c}\text { GenBank accession no. } \\
\text { for ITS1-5.8S-ITS2 region }\end{array}$ \\
\hline
\end{tabular}

Results of BLAS analysis concluded that; the fungus was $F$. proliferatum based on the high identity value of (96\%) and (0) E-value. Used BLAST search tool, to compare the resulting sequences with sequences of rDNA accessed at GenBank, the phylogenetic analysis for $F$. proliferatum showed that the sbtained sequences share $96 \%$ homology to Fusarium proliferatum strains: Iranian isolate (MH547040), Bangladesh strain (MK446840), Pakistani strain (MK583509), Tunisian strain (MH329789), Indian strain (MK751710), and Chinese strain (MK158224), (Figure 3) and Table (5). Together, morphological and molecular identification showed that Fusarium isolate was F. proliferatum, GenBank accession no. is (MK112619). This isolate was aligned with six accession sequences of $F$. proliferatum. The distance scale between sequences is $0.05 \%$, whereas 0.041 is the ideal tree with the branch length sum. As shown in (Figure 3), some of the branches have elevated bootstrap values. The phylogenetic tree divided all species into two clades. Our isolate showed a separate clade.

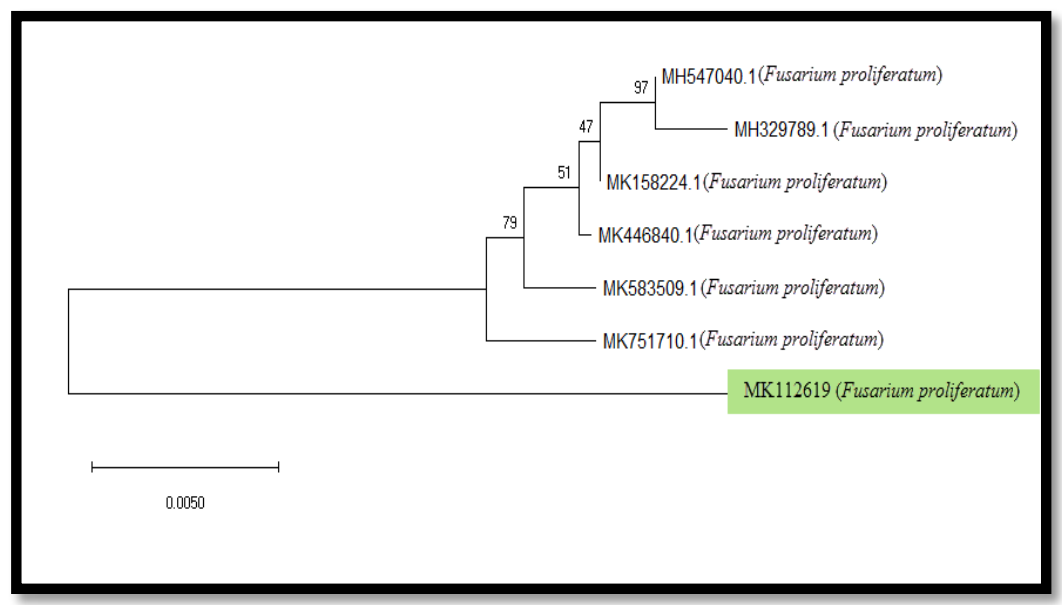

Figure 3: Phylogenetic tree of $F$. proliferatum based on the nucleotide sequence of ITS-rDNA. The values on the branch represent the bootstrap values.

Kurdistan Journal of Applied Research | 3rd International Conference on Health \& Medical Sciences: Insight into Advanced Medical Research (ICHMS 2019)| 134 
Table 5: Phylogenetic tree of $F$. proliferatum depending on the nucleotide sequence of ITS-rDNA.

\begin{tabular}{|c|c|c|c|c|}
\hline No. & Isolate Species & GenBank Acc. No. & $\begin{array}{c}\text { Geographic } \\
\text { Location }\end{array}$ & Similarity \\
\hline 1. & F. proliferatum & (MH547040), & Iran & $\mathbf{9 6 \%}$ \\
\hline 2. & F. proliferatum & (MK446840) & Bangladesh & $\mathbf{9 6 \%}$ \\
\hline 3. & F. proliferatum & (MK583509) & Pakistan & $\mathbf{9 6 \%}$ \\
\hline 4. & F. proliferatum & (MH329789) & Tunisia & $\mathbf{9 6 \%}$ \\
\hline 5. & F. proliferatum & (MK751710) & India & $\mathbf{9 6 \%}$ \\
\hline 6. & F. proliferatum & (MK158224) & China & $\mathbf{9 6 \%}$ \\
\hline
\end{tabular}

\section{DISCUSSION}

Mycological examination in this case study detected positive result for both direct $\mathrm{KOH}$ and culture of $F$. proliferatum in the left first toenail of the suspected case. In the present study, Fproliferatum represented a common etiological agent of toenail onychomycosis. In previous studies in Iraq, Fusarium species is also recorded from nail infection particularly by not by molecular method, only by routine and conventional morphological approach. And this species of Fusarium may be replacing other mold fungi such as Aspergillus sp., Scopulariopsis brevicaulis, Scytalidium dimidiatum [11,12], Fusarium spp., Scytalidium hyalinum and, recently, Onychocola canadensis $[8,9,10]$, as an etiology for toe nail onychomycosis in Iraq with much lower frequency than reported previously. The PCR-based technique is reliable for most typing purpose. Recently, sequencing of ITS region is the golden typical for delineation of dermatophyte species [30,31]. Therefore, in a recent investigation, a molecular approach was employed to confirm the identification of the Fusarium strain isolated formerly by culture and classical methods from patients with chronic onychomycosis. DNA extracted using solid phase method after dipping in liquid nitrogen followed by PCR amplification of ITS region of ribosomal DNA with pair of diverse primers. In the present study, the fact which observed during the ITS-PCR amplification was differ from the previous studies, as expected, size of PCR products from fungal species with different base pair length, (Figure 4) that the pattern band was 471 bp for this species of Fusarium on the gel during the electrophoretic analysis for genus. The difference refer to a genetic alteration in the ITS region or false diagnosis. Consequently, the investigation of ITS region by sequencing method was provided with a simple technique for fungal characterization [32]. The diverse length of ITS region among fungal species confirmed the results of laboratory conventional identification [33,34].

The finding PCR product was sequenced according to Macrogen company/ Korea using one primer ITS1 and our sequence was placed in GenBank with accession no. MK112619 (ITS). For phylogenetic analysis, sequences of the amplified product was retrieved from GenBank in FASTA format. Neighbor-Joining (NJ) technique for phylogenetic analysis [28]. It was accomplished with the MEGA v. X software [35]. The phylogenetic tree was built by Pdistance model Kimura [36] with the 'pairwise deletion of gaps option'. A nucleotide BLAST search with $471 \mathrm{bp}$ and indicated an extreme homology of $96 \%$ with $F$. proliferatum of Iranian isolate (MH547040), Bangladesh strain (MK446840), Pakistani strain (MK583509), Tunisian strain (MH329789), Indian strain(MK751710), and Chinese strain (MK158224),Table(5).The isolate MK112619.1 (S10) clustered with several accession numbers (mentioned previously) in a common ancestor, but with the supporting of different bootstraps (Figure 3).The low distance between the different accessions indicated that the variations among the accessions were low. The comparison between our isolate and the ITS reference sequences that were obtained from the NCBI database confirmed the identification of isolates at genus and species level. 


\section{CONCLUSION}

In this study, we achieve that there is an epidemiological alteration regarding the etiological agent in our region. Fusarium proliferatum also may be replacing other pathogenic molds as an etiology of toenail onychomycosis among Iraqi patients. We therefore found that the molecular assessment of the fungal ribosomal DNA region of ITS is essential for the best drug choice and is a straightforward, quick and reproducible instrument during the identification of fungal species in the laboratory.

\section{REFERENCE}

[1] A. Banu, M. Anand, L. Eswari,” A rare case of onychomycosis in all 10 fingers of an immunocompetent patient”, Indian Dermatol Online J.;4(4):302-4, 2013. doi:10 /2229-5178.120649.

[2] E. Haneke, D. Roseeuw ,” The scope of onychomycosis: epidemiology and clinical features”, Int J Dermatol 38 Suppl 2: 7-12,1999.

[3] Y. Ogasawara, "Prevalence and patient's consciousness of tinea pedis and onychomycosis”, Nihon Ishinkin Gakkai Zasshi 44: 253-260,2003.

[4] MA. Ghannoum, RA. Hajjeh, R. Scher, N. Konnikov, AK. Gupta, et al, “A large-scale North American study of fungal isolates from nails: the frequency of onychomycosis, fungal distribution, and antifungal susceptibility patterns”, J A Acad Dermatol 43: 641-648, .2000.

[5] RK. Scher, "Onychomycosis is more than a cosmetic problem. Br J Dermatol 130: 15,1994.

[6] MA. Ghannoum, N. Isham , “Fungal Nail Infections (Onychomycosis): A Never-Ending Story?”, PLoS Pathogens 10(6): , 2014.

[7] S. Ahuja, S. Malhotra, H. Charoo,” Etiological agents of onychomycosis from a tertiary care hospital in central Delhi”, India. Indian J Fundam Appl Life Sci;1:11-4 ,2011.

[8] DW. Denning, EGV. Evans,CC. Kibbler, MD. Richardson, MM. Roberts, TR. Rogers, et al. ,’Fungal nail diseases: a guide to good practice" (report or a working group of the British society for medical mycology). BMJ; 311: 1277-81, 1995.

[9] AK. Gupta, CB. Horgan-Bell, RC. Summerbell, “Onychomycosis associated with Onychocola canadensis: Ten case reports and review of the literature”, J Am Acad Dermatol; 39: 410-7, 1998.

[10] AK. Gupta, EA. Cooper, P. MacDonald, RC. Summerbell, "Utility of inoculum counting (Walshe and English Criteria) in clinical diagnosis of onychomycosis caused by nondermatophyte filamentous fungi',J Clin Microbiol ; 39(6): 2115-21, 2001.

[11] AK. Gupta, JE. Ryder, R. Baran, RC. Summerbell, “Nondermatophyte onychomycosis”, Dermatol Clin; 21: 257-68, 2003.

[12] DH. Ellis, JE. Marley, AB. Watson, TG. Williams, "Significance of non-dermatophyte molds and yeasts in onychomycosis”,Dermatology; 194(Suppl 1): 40-2,1997.

[13] T. J. Walsh, and A. H. Groll, "Emerging fungal pathogens: evolving challenges to immunocompromised patients for the twenty-first century”, Transpl. Infect. Dis.1:247-26, 1999.

[14] M. Nucci, and E. Anaissie, "Cutaneous infection by Fusarium species in health and immunocompromised hosts: implications for diagnosis and management”, Clin. Infect. Dis. 35:909-920, 2002.

[15] N. Hattori, A. Shirai, Y. Sugiura, W. Li, K. Yokoyama, Y. Misawa, K. Okuzumi, and K. Tamaki, “Onychomycosis caused by Fusarium proliferatum”,Br. J. Dermatol.153:647-649, 2005.

[16] C. Ferrer, J. Alio, A. Rodriguez, M. Andreu, and F. Colom, "Endophthalmitis caused by Fusarium proliferatum', J. Clin. Microbiol. 43:5372-5375, 2005.

[17] R. V. Fleming, T. J. Walsh, and E. J. Anaissie, “Emerging and less common fungal pathogens”, Infect. Dis. Clin. North Am. 16:915-933, 2002.

[18] R. Ray, M. Ghosh, M. Chatterjee, N. Chatterjee,M. Banerjee, “Onychomycosis Caused by Fusarium dimerum“, J Clin Sci Res, 5:44-8,2016.

[19] GF. Orr, HH. Kuehn, OA Plunkett, “A new genus of the Gymnoascaceae with swollen peridial septa”, Can J Bot 41:1439- 1456. doi:10.1139/b63-126, 1963.

[20] J. Kane, R. C. Summerbell, L. Sigler, S. Krajden, \& G. Land, Laboratory handbook of dermatophytes. Belmont CA: Star Piblishers, 1997.

[21] I. Weitzman, \& R. Summerbell, “The dermatophytes”, Microbiology Review, 8 (2), 240-259, 1995.

[22] B. L. Hainer, “Dermatophyte Infections”, American family physician. 67(1):101-108, 2003.

[23] G.Rebell \& D. Taplin, “ Dermatophytes; their recognition and identification”, University of Miami Press, Coral Gables, Florida, 1970.

[24] K. Makimura, Y. Tamura, T. Mochizuki, A. Hasegawa , Y. Tajiri, R. Hanazawa, et al., Phylogenetic classification and species identification of dermatophyte strains based on DNA sequences of nuclear ribosomal internal transcribed spacer 1 regions”, J Clin Microbiol, 37 ,pp. 920-924, 1999.

[25] R. B. Corley, A guide to methods in the biomedical sciences. New York:Springer, 2005. 
[26] P. Desjardins, D. Conklin ,’NanoDrop Microvolume Quantitation of Nucleic Acids. JoVE. 45, 2010. http://www.jove.com/index/Details.

[27] S. I. Ismail,” Morphological and molecular identification of dermatophytes isolated from patients in Erbil City”, M.Sc.Thesis. College of Science. Salahaddin University. Erbil-Iraq, 2015.

[28] N. Saitou, M. Nei, "The neighbor-joining method: a new method for reconstructing phylogenetic trees", Molecular Biology and Evolution 4: 406-425, 1987.

[29] S. Kumar , Li M. Stecher, C. Knyaz, and K.Tamura, "MEGA X: Molecular Evolutionary Genetics Analysis across computing platforms “, Molecular Biology and Evolution 35:1547-1549,2018.

[30] H. C. Li, J. P. Bouchara, M. M.Hsu, R. Barton, S. Su, \& T. C. Chang , "Identification of dermatophytes by sequence analysis of the of the rDNA gene internal transcribed spacer regions”, J. Med Microbial, 57, 592-600, 2008.

[31] P. Nenoff \& U. F. Haustein , “Tinea corporis due to Trichophyton tonsurans”, Malmsten-report of a patient from Zaire. J. Mycosis, 40 (3-4), 127-129, 1997.

[32] C. J. Jackson, Barton, R. C., \& Evans, G. V, "Species identification and strain differentiation of Dermatophyte fungi by analysis of Ribosomal- DNA intergenic spacer regions”, Journal of Clinical Microbiology, 37 (4), 931 936,1999.

[33] B. Ahmadi, H. Mirhendi, , M. R. Shidfar, S. Nouripour-Sisakht, N. Jalalizand, , M. Geramishoar, , \& G. R. Shokoohi, “A comparative study on morphological versus molecular identification of dermatophyte isolates”, J Mycol Med, 25 (1), 29-35, 2015.

[34] K. Al-Khafajii ,'Myco epidemiologic and genetic study of rermatophytosis an non dermatophytes in middle euphrates Iraq", African Journal of Microbiology Research, 8 (24), 2381-2386, 2014.

[35] K. Tamura, D. Peterson, N. Peterson, G. Stecher, M. Nei, S. Kumar, MEGA5: "Molecular evolutionary genetics analysis using maximum likelihood evolutionary distance, and maximum parsimony methods”, Molecular Biology and Evolution 28: 2731-2739, 2011.

[36] M. Kimura, “A simple method for estimating evolutionary rates of basesubstitutions through comparative studies of nucleotide sequences”, Journal of Molecular Evolution 16: 111-120, 1980. 